\title{
Evaluating Visual Search Performance with a Multi Layer Display
}

\author{
Andreas Dünser \\ HIT Lab NZ, University of \\ Canterbury, New Zealand \\ andreas.duenser@hitlabnz.org
}

\author{
Mark Billinghurst \\ HIT Lab NZ, University of \\ Canterbury, New Zealand \\ mark.billinghurst@hitlabnz.org
}

\author{
Gabriela Mancero \\ Middlesex University, The \\ Burroughs, London, UK \\ g.mancero@mdx.ac.uk
}

\begin{abstract}
A Multi Layer Display (MLD) is a novel device which allows content to be shown on different depth planes. Earlier research indicates that stereoscopic depth information can be beneficial in visual search tasks. This has not yet been explored for this new display technology. In this paper we present the results of an experiment to explore how the actual depth information afforded by a two layer MLD affects visual search task performance. We found that placing distracters and targets on different depth layers can significantly improve performance in complex search tasks. We discuss these findings and provide suggestions on how to arrange the stimuli across the two layers in order to get the full benefit of the depth information.
\end{abstract}

\section{Categories and Subject Descriptors}

B.4.2 Hardware: Input/Output Devices; H.5.2 Information interfaces and presentation (e.g., HCI): User Interfaces

\section{General Terms}

Performance, Experimentation, Human Factors

\section{Keywords}

Multi layer display, visual search, depth perception

\section{INTRODUCTION}

A Multi Layer Display (MLD) [6], is a unique device in which two or more LCD displays are stacked on top of each other separated by a transparent layer. For example, figure 1 shows a two layer display which allows visual information to be presented on two different physical layers. Information that is displayed on the back screen is visible through the front screen. Compared to other technologies that use stereoscopic depth cues, MLDs show actual depth created by the physical separation of the two screens.

Although there are a variety of potential applications, there needs to be a better understanding of the unique display properties of MLDs and how they relate to human perception.

OZCHI 2008, December 8-12, 2008, Cairns, QLD, Australia.

Copyright the author(s) and CHISIG.

Additional copies are available at the ACM Digital Library (http://portal.acm.org/dl.cfm) or can be ordered from

CHISIG(secretary@chisig.org)

OZCHI 2008 Proceedings ISBN: 0-9803063-4-5
More specifically, we need to understand how the visual depth created by the overlapping LCDs affects user performance, and therefore how users can benefit from showing information across multiple layers in depth. Although human perception has been comprehensively studied with interfaces using stereoscopic depth or other simulated depth cues, few studies have explored such issues with actual depth.

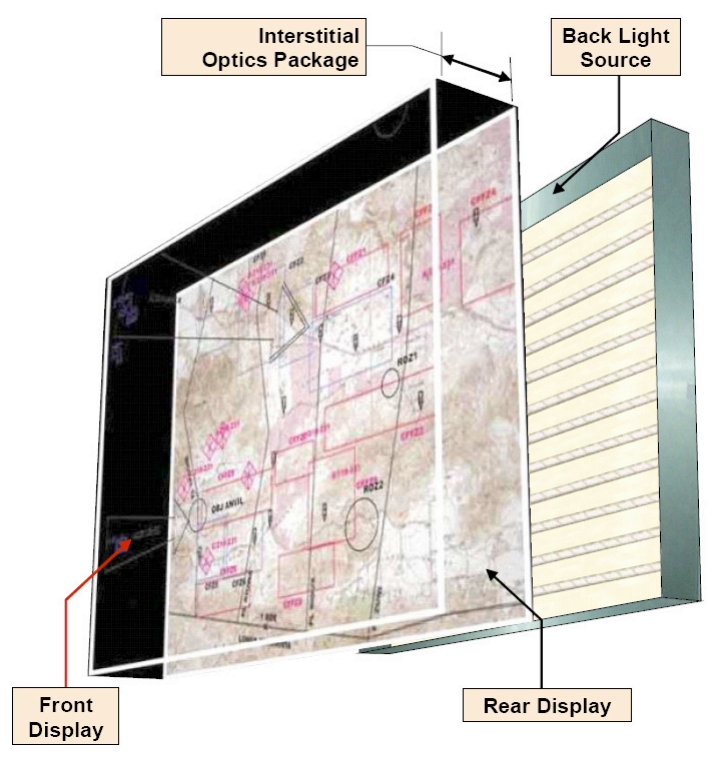

Figure 1. Construction of a Multi Layer Display [6]

In our research we reviewed work from different fields (e.g. $\mathrm{CHI}$, human factors, and cognitive psychology), particularly in the areas of visual search, vigilance, alerting, and target discrimination, to study how the depth information may affect performance. We identified visual search as an important task in fields such as gaming, medicine, air traffic control, emergency dispatch, and engineering, where operators have to deal with visually complex scenarios and quickly and reliably extract relevant information.

The affordances of a MLD [9] imply that the it has the potential to improve various aspects of visual information search and detection. However, only some of these affordances have been explored with formal user studies. In general this earlier research suggested that depth information can be valuable in complex search tasks. However, these areas have not been explored in detail with a MLD.

An advantage of a MLD is that for the user to perceive depth, no additional apparatus is needed such as glasses (e.g. polarised, 
coloured) or mirror setups to separate views. This can be an important factor for certain tasks or workplace setups where the use of such additional equipment is either inconvenient or can hinder task execution. Thus it seems important to review related research and evaluate the depth display properties of MLDs and their effect on human perception in more detail.

\section{RELATED WORK}

\subsection{Multi Layer Displays}

Aboelsaadat and Balakrishnan [1] investigated issues surrounding interference in the context of one layer versus two layer displays with a Stroop test. They found that performance using two layer displays degrades when the stimuli compete for user attention. For non-spatially overlapping stimuli, performance is dependent on the assignment of stimuli to various layers, with the single layer display equalling or outperforming the two layer display in all cases.

As Wickens and Hollands [8] point out, in displays designed to facilitate parallel processing it is sometimes difficult to narrow the focus of attention and shut out unwanted inputs, in this case the distracting stimuli. However, depth cues can be used to assist with focusing on relevant information. They found that search times were shorter for targets separated in depth. This suggests that detection times can be improved if a target and distracter can be separated in depth. The experiment of Hayes, Wong, and Moores [3] indicates that the MLD can offer benefits in helping users to focus on relevant information, and reducing visual clutter while still retaining all the information necessary for maintaining awareness of the overall situation.

Wong et al. [10] explored the effectiveness of using depth and alpha-blending with varying levels of transparency to create a sense of visual depth, while comparing objects presented on both layers of a two layer MLD to a control condition using a Single Layer Display (SLD). They found that under easy task conditions there was no difference in performance between MLD and SLD conditions. However, under more complex conditions, such as the need to perform cognitively more demanding comparisons, the MLD showed significant improvements over the SLD.

\subsection{Visual search}

Search for a single feature is conducted in parallel [7] and search times are independent of the number of stimuli presented on the screen. In conjunction searches the search for targets is based on differences in more than one dimension (e.g. colour and shape). If the number of distracters is increased a corresponding increase in search time can be observed as people have to serially search the display.

Results from Nakayama and Silverman [5] suggest that depth might play a different role in visual search compared to other visual features. The visual system might use depth to segregate the visual array into depth planes. In their experiments they found that conjunctive tasks combining stereoscopic disparity with either colour or motion were qualitatively different from motion-colour searches. The observers had the distinct impression that each plane could be searched effortlessly. Correspondingly, the reaction time functions for each of these searches were constant over set sizes. This implies that the visual system can perform a parallel search in one depth plane without interference from target-like distracters in another depth plane.

Moore et. al. [4] replicated Nakayama and Silverman's findings of efficient search performance for a depth and colour conjunction search. De la Rosa et al. [2] investigated perceptual grouping in conjunction searches and compared search performance of conjunctive searches (stereoscopic depth and colour) using two different search displays (consisting of two or six depth planes). Response times across display conditions and set sizes did not differ significantly. Results of this study imply that conjunctive search using stereoscopic depth is more effortless.

This research shows that stereoscopic depth can improve search performance in more complex search tasks. In the next section we present a study to investigate if and how different stimulus arrangements on a two layer MLD affect human performance in visual search tasks.

\section{VISUAL SEARCH EXPERIMENT}

\subsection{Participants}

Twenty university students ( 8 female, 12 male) aged between 18 and 46 years participated in the study. All participants had normal or corrected to normal vision. Outliers (values of any variable more than $1.5^{*} \mathrm{IQR}$ (Interquartile Range)) were not included in the analysis.

\subsection{Materials}

Equipment: Stimuli were presented on a 17 inch two layer MLD set at a resolution of 1024 x 768 (per screen). An X-keys USB programmable keyboard (20-keys) was used for input.

Stimuli: The stimuli were targets and distracters 30x30 pixels in size which differed in colour (red, blue), shape (circle, triangle), and depth (front, back layer) or a combination of these features (see Figure 2). The number of stimuli varied between 15,30 , and 45 objects and were presented in such a way that they did not overlap each other. The positions of the targets and distracters on the display were generated randomly.
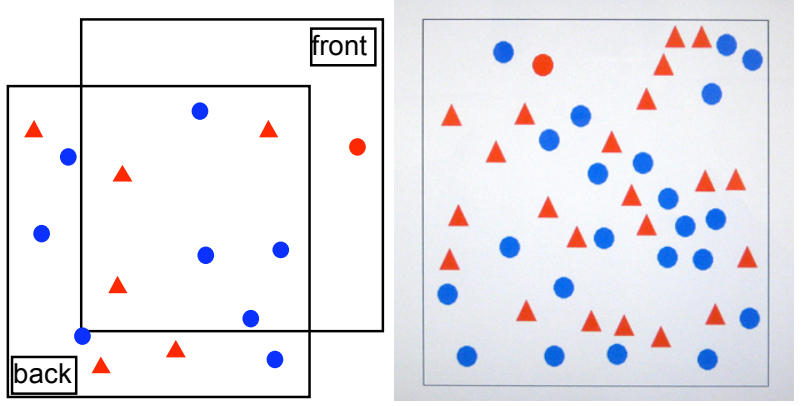

Figure 2. Left: stylized example for a colour + shape + depth conjunction search (only the target - the red circle - is on the front layer); Right: actual screenshot

\subsection{Design}

The user's task in the experiments was to detect a single red circle in all conditions. The single search conditions were colour (single layer; all stimuli were shown on the MLDs front layer; distracters: blue circles), shape (single layer, distracters: red triangles), and depth (target on the front layer; distracters 
(red circles) on the back layer). For the conjunction searches different feature combinations were realized:

- colour + shape (cs): blue circles, red triangles, and target (red circle) all shown on the front layer

- colour + depth:

- red circles on the back layer; blue circles and target (red circle) on the front layer - $(c d)$

- red and blue circles on the back layer; target (red circle) on the front layer $-(c d(t f))$

- shape + depth:

- red circles on the back layer; red triangles and target (red circle) on the front layer ( $\mathrm{sd}$ )

- red circles and triangles on the back layer; target (red circle) on the front layer- $(\mathrm{sd}(\mathrm{tf}))$

- colour + shape + depth (csd): red circles, red triangles and blue circles on the back layer; target (red circle) on the front layer

Each of these conditions included a block of 15 practice trials and a block of 60 actual trials. Whether the target was present or not and the set size $(15,30,45$ stimuli) were manipulated pseudo randomly within the blocks (balanced number of 'stimulus present' and set sizes across the blocks). Thus for each condition and set size the participants responded to 10 trials with and 10 trials without the stimulus which resulted in 540 data points overall per subject.

The participants were told to work as fast as possible and at the same time to make as few errors as possible. They were asked to press a specific button if they thought the target was present and another button for not present. We used reaction times and the percentage of correct answers as experimental metrics.

\subsection{Results}

The first step was to compare the slopes of the reaction times for the different conditions (see Figure 3 ). The colour only condition $(c)$ showed the flattest slope and the shape + depth $(s d)$ condition the steepest. Reaction times in the $s d$ condition rose by $16.65 \mathrm{msec}$ with each additional item in the search display whereas in the $c$ condition they stayed practically the same.

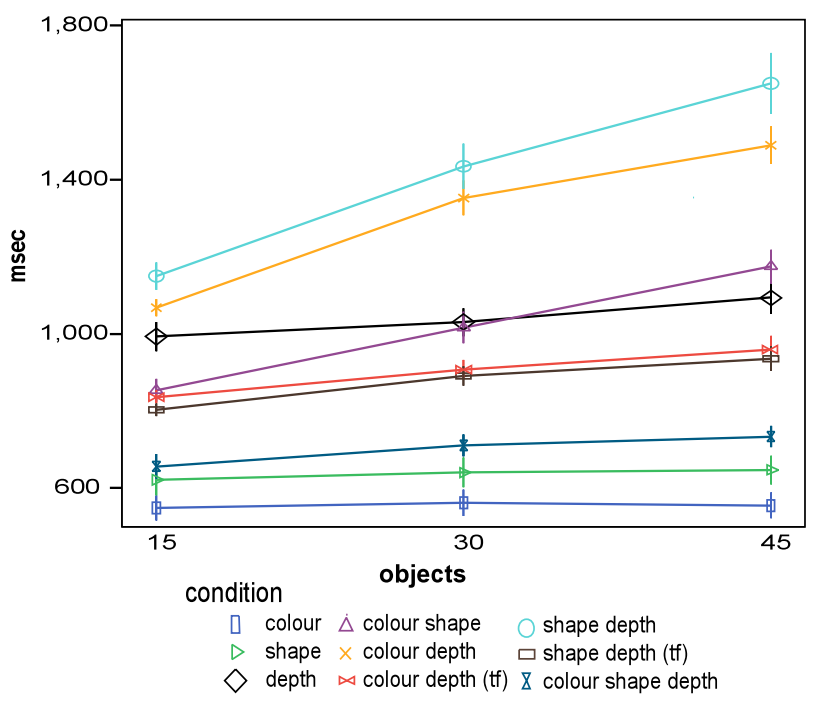

Figure 3. Reaction times for search task (error bars +/- SE)
Slopes were compared with pair wise T-tests (with Bonferroni adjustment). The slopes of all single searches differed significantly from the 'colour depth' $(c d)$ and 'shape depth' $(s d)$ slopes (all $p<.05$ ). The $c$ and $s$ conditions also differed from 'colour shape' $(c s)(p<.01)$. The slopes of conjunction searches where only the target was in the front layer were relatively flat and did not differ from the slopes of the single search conditions. The conditions with only the target in the front layer ('colour depth' $(c d(t f))$ and 'shape depth' $(s d(t f)))$ each had significantly flatter slopes than their counterparts in which also distracters were in the front $(p<.05)$.

For the reaction times (see Table 1$)$ a 9 (conditions) by $3(15$, 30, 45 objects) within subjects ANOVA was computed. The main effects (condition: $F_{3.80,53.13}=47.37, p<.01$; number of objects: $\left.\quad F_{1.51,21.12}=98.10, \quad p<.01\right)$ and the interaction $\left(F_{10.49,146.83}=17.00, p<.01\right)$ showed significant results. In condition $c$ participants showed the fastest overall reaction time and the post hoc analysis ${ }^{1}$ showed that this condition differed significantly from all the others. The two conjunction searches with distracters on both the back and the front layers ( $c d$ and $s d$ ) showed the longest reaction times. In the same search conditions ( $t f)$ with only the target on the front layer, participants reacted significantly faster. Search times in the $t f$ conditions were slightly but not significantly higher than the $c$ and $s$ single searches and even slightly but again not significantly lower than search times for the single depth condition. Depth only and cs conditions showed similar mean search times. The $d$ condition was only faster than the $s d$ condition.

The mean search time for the combination of all three stimulus dimensions $c s d$ was similar to that of the $s$ condition and significantly different from all the others. Only in the $c$ condition did the participants reacted significantly faster.

Post hoc comparisons for the number of objects showed that mean reaction times for 15 objects $(M=851.03 ; S D=26.74)$, $30(M=926.94 ; S D=35.31)$ and $45(M=999.02 ; S D=40.58)$ all differed significantly from each other $(p<.01)$.

Table 1. Mean reaction times in milliseconds

\begin{tabular}{|c|c|c|c|c|}
\hline & \multicolumn{3}{|c|}{ Objects } & \multirow[b]{2}{*}{ total } \\
\hline & 15 & 30 & 45 & \\
\hline colour & 529 & 543 & 534 & 535 \\
\hline shape & 626 & 642 & 646 & 638 \\
\hline depth & 981 & 1028 & 1100 & 1036 \\
\hline colour shape & 838 & 1026 & 1182 & 1015 \\
\hline colour depth & 1054 & 1297 & 1431 & 1261 \\
\hline colour depth (tf) & 802 & 860 & 899 & 854 \\
\hline shape depth & 1126 & 1378 & 1560 & 1354 \\
\hline shape depth (tf) & 777 & 860 & 917 & 851 \\
\hline colour shape depth & 656 & 708 & 723 & 696 \\
\hline total & 821 & 927 & 999 & \\
\hline
\end{tabular}

\footnotetext{
${ }^{1}$ Unless stated, Bonferroni adjustment was used in all post hoc multiple comparisons
} 
As a measure for accuracy the percentage of correct answers for each condition was analysed. Accuracy was significantly different between the conditions $\left(F_{5.04,70.55}=6.88, p<.01\right)$. The percentage of correct answers was lowest in the $c d$ condition $(95.67 \%)$ which differed significantly $(p<.05)$ from the relatively high percentages in conditions $c d(t f)(99.22 \%)$ and csd $(99.22 \%)$.

\section{DISCUSSION}

In this experiment we explored if the depth-information provided by the MLD could improve performance in visual search tasks. This assumption was partially substantiated. In conjunction searches the depth information improved performance. However, one has to be careful how to arrange the stimuli. We found that putting only the target on the front layer allows the users to perform the search task more effectively.

Reaction times for the colour and shape only conditions stayed almost the same when the number of distracters in the display was increased. There was a small increase in reaction time in the depth only condition (3.39 msec for each additional stimulus) but the slope was quite flat. This shows that participants performed parallel searches in the single search conditions.

The colour-shape conjunction search and the colour-depth and shape-depth search conditions with distracters in the front and back layer showed much steeper slopes. This indicates that the participants were performing serial searches in these conditions. However, if distracters were presented on the back layer and only the target on the front, the slopes were flatter. Thus we could replicate Nakayama and Silvermans' findings [5] for the conditions with only the target on the front layer. Even in the condition with three stimulus dimensions (colour, shape and depth) the slope was quite flat. The participants obviously did not have to search the whole display in a serial search. Alternatively, having distracters on both layers, which is similar to Nakayama and Silverman's experimental setup [5], did not lead to a parallel search. Displaying only the target on the front layer and the distracters on the back seems to make the target more salient [8] and hence easier to detect.

Colour and shape single searches could be performed relatively quickly. However, in the depth single search condition reaction times were higher and similar to a colour and shape conjunction search. In single searches (only one stimulus dimension) colour or shape seems to help the participants better to distinguish the target from distracters. The participants also searched efficiently when all the stimulus dimensions of colour, shape and depth were combined. Search times in this condition were similar to shape only searches and faster than all except the colour-only condition.

Search times in conjunction searches with distracters in both layers were high. Reaction times in these conjunction searches were significantly faster when only the target was displayed on the front screen. However, it is interesting that participants showed higher reaction times in the depth only condition compared to the $c s d$ condition. In both conditions only one stimulus (the target) was on the front layer. Having distracters that differed from the target not only in depth but also colour and shape seemed to make the search task easier.
The conditions with only the target on the front layer produced results with a relatively high accuracy. Thus, compared to having distracters in both layers, putting just the target on the front layer and distracters on the back layer seemed to help the participants to judge more accurately whether the target was present or not.

\section{CONCLUSION}

Our results show that using the depth information provided by a MLD can increase search performance in relatively complex searches. This suggests that the depth information helps users to visually distinguish the target from the distracting stimuli. This indicates that the depth afforded by MLDs can support users in visually complex environments. We could replicate the results of prior studies that used stereoscopic displays for search tasks. Hence the MLD presents itself as feasible option for these types of tasks in various application areas, especially because there is no need for additional equipment to perceive depth.

We provided initial evidence about how the actual depth display properties of MLDs can influence human perception but further research is needed. Future studies might involve similar experimental setups using other stimulus combinations not included in our studies.

\section{REFERENCES}

1. Aboelsaadat, W., and Balakrishnan, R. An empirical comparison of transparency on one and two layer displays. In Proc. HCI 2004, (2004), 53-67.

2. de la Rosa, S., Moraglia, G., and Schneider, B. Search behavior in conjunctive visual searches with stereoscopic depth and color. Poster presented at the European Conference for Visual Perception. (2005).

3. Hayes, J., Wong, W., and Moore, A. Information layering to de-clutter displays for emergency ambulance dispatch. In Proc. 13th European Conference of Cognitive Ergonomics ECCE13, ETHZ Press. (2006).

4. Moore, A. A tangible augmented reality interface to tiled street maps and its usability testing. In Proc. 12th International Symposium on Spatial Data Handling, (2006).

5. Nakayama, K., and Silverman, G.H. Serial and parallel processing of visual feature conjunctions. Nature, 320, 20 (1986), 264-265.

6. PureDepth. (2007). WHITE PAPER Multi-Layer Displays http://www.puredepth.com/Docs_Downloads/HardwareWhi tePaper_Feb_2007.pdf

7. Treisman, A. Search, similarity, and integration of features between and within dimensions. Journal of Experimental Psychology: Human Perception \& Performance, 17, 3 (1991), 652-676.

8. Wickens, D., and Hollands, J. Engineering Psychology and Human Performance ( $3^{\text {rd }}$ ed.). Prentice Hall,Upper Saddle River, NJ, 2000.

9. Wong, W. (2003). Technology affordances of the ActualDepth(tm) multi-layered display: TBG Grant Ref. Num. DVIL 0201: Human-Computer Interaction of the ActualDepth(tm) 3-D Display Technology.

10. Wong, W., Joyekurun, R., Mansour, H., Amaldi, P., Nees, A., and Villanueva, R. Depth, layering and transparency: developing design techniques. In Proc. OzCHI '06, Canberra, Australia, CHISIG Australia, (2005), 1-10. 